\title{
Doxorubicin Delivered Using Nanoparticles Camouflaged with Mesenchymal Stem Cell Membranes to Treat Colon Cancer
}

This article was published in the following Dove Press journal: International Journal of Nanomedicine

\author{
Yi Liu (D)' \\ Jingtong Zhao ${ }^{2}$ \\ Jinlan Jiang ${ }^{2}$ \\ Fangfang Chen ${ }^{1,3,4}$ \\ Xuedong Fang ${ }^{1,3}$ \\ 'Department of Gastrointestinal \\ Colorectal and Anal Surgery, China-Japan \\ Union Hospital, Jilin University, \\ Changchun, jilin 130033, People's \\ Republic of China; ${ }^{2}$ Department of \\ Central Laboratory, China-Japan Union \\ Hospital, Jilin University, Changchun, Jilin \\ 130033, People's Republic of China; \\ ${ }^{3}$ State Key Laboratory of Inorganic \\ Synthesis and Preparative Chemistry, \\ College of Chemistry, Jilin University, \\ Changchun, Jilin I300/2, People's \\ Republic of China; ${ }^{4}$ Key Laboratory of \\ Zoonoses Research, Ministry of \\ Education, Jilin University, Changchun, \\ Jilin 130062, People's Republic of China
}

Correspondence: Xuedong Fang; Fangang Chen

Email fangxd1961@163.com;

cffemail@I63.com
Purpose: The primary goal of the present study was to design doxorubicin (DOX)-loaded superparamagnetic iron oxide (SPIO) nanoparticles (NPs) coated with mesenchymal stem cell (MSC) membranes and explore their effect on colon cancer in vitro and in vivo.

Methods: DOX-SPIO NPs were coated with MSC membranes using an extruder, and the morphological characteristics of MSC membrane-camouflaged nanodrug (DOX-SPIO@MSCs) evaluated by transmission electron microscopy (TEM) and NP-tracking analysis. Drug loading and $\mathrm{pH}$ response were assessed by UV spectrophotometry. Intracellular colocalization was analyzed using NP-treated MC38 cells stained with 3,3'-dioctadecyloxacarbocyanine perchlorate and Hoechst 33342. Cellular uptake was analyzed using an inverted fluorescence microscope and flow cytometry and cytotoxicity evaluated by cell counting kit- 8 assay. Biological compatibility was assessed by hemolysis analysis, immunoactivation test and leukocyte uptake experiments. Furthermore, intravenous injection of chemotherapy drugs into MC38 tumor-bearing C57BL/6 mice was used to study anti-tumor effects.

Results: Typical core-shell NP structures were observed by TEM. Particle size remained stable in fetal bovine serum and phosphate-buffered saline (PBS). Compared with DOXSPIO, DOX-SPIO@MSCs improved cellular uptake efficiency, enhanced anti-tumor effects, and reduced the immune system response. Animal experiments demonstrated that DOXSPIO@MSCs enhanced tumor treatment efficacy while reducing systemic side effects.

Conclusion: Our experimental results demonstrate that DOX-SPIO@MSCs are a promising targeted nanocarrier for application in treatment of colon cancer.

Keywords: iron oxide, mesenchymal stem cells, doxorubicin, colon cancer

\section{Introduction}

Nanocarriers based on targeted drug delivery systems have been extensively researched and rapidly developed for application in clinical oncology treatments. ${ }^{1-4}$ To date, enhanced permeability and retention effects remain the primary mechanism of uptake of tumor-specific nanocarriers. ${ }^{5}$ Most nanoparticles (NPs) are recognized by the immune system and cleared as foreign substances, thereby limiting their clinical application. ${ }^{6}$ Hence, there is an urgent need to develop safer and more productive approaches. Biomimetic NPs decorated with the bioactive membranes have become one of the most appealing structures in this context, because the variability of NPs combined with the complexity and functionality of cell membranes generates high adaptability to the tumor microenvironment. ${ }^{7,8}$ For example, Zhang and coauthors developed cell membrane-coated nanorods by ultrasonic mechanical mixing, and the 
resulting formulation exhibited improved tumor recognition and exhibited highly efficient endocytosis. ${ }^{9}$

Mesenchymal stem cells (MSCs) are a class of multipotent cells able to self-renew and with potential to differentiate into multiple cell lineages. In addition, MSCs exhibit unique advantageous characteristics, including a lack of immunogenicity, ${ }^{10}$ ability to circulate in the blood for extended periods, ${ }^{11}$ and tumor/inflammatoryspecific properties ${ }^{12}$ in vivo. As a promising source of cell membranes, MSCs can be isolated from various tissues and increased in quantity in the laboratory. ${ }^{13}$ Further, MSC-coated NPs can decrease macrophage uptake, to reduce clearance by the reticuloendothelial system, and increase targeted cellular uptake and tumor-selective accumulation. ${ }^{14}$ Use of MSC membranes for surface modification of functional NPs to develop biomimetic drug delivery platforms has become an area of intense research focus. ${ }^{15,16}$

Inspired by the remarkable performance of MSCs in previous investigations, in this study, we used MSC membrane coating as a strategy to camouflage a nanodrug for treatment of colon cancer. Doxorubicin (DOX) superparamagnetic iron oxide (SPIO) NPs coated with mesenchymal stem cell (MSC) membranes (DOX-SPIO@MSCs) NPs exhibited better tumor cellular uptake, invoked decreased immune responses, and potentiated strong antitumor effects, with minimal adverse effects. Our results demonstrate that DOX-SPIO@MSCs provide an excellent nanoplatform for engineering tumor-targeting drug delivery systems.

\section{Materials and Methods}

\section{Materials and Cell Lines}

Dextran-coated SPIO (20 kDa), consisting of multiple crystalline iron oxide cores arranged into wormlike strings, with a hydrodynamic diameter of $76.6 \pm 1.6 \mathrm{~nm}$ (Figure S1), was donated by Professor Dmitri Simberg, University of Colorado Anschutz Medical Campus, USA. Phosphatebuffered saline (PBS), penicillin-streptomycin and $\alpha$ Minimum Essential Medium ( $\alpha$-MEM) were all obtained from HyClone Laboratories (Logan, Utah, USA). Dulbecco's Modified Eagle's Medium (DMEM), 0.25\% Trypsin-EDTA, and fetal bovine serum (FBS) were from Gibco Life Technologies (Grand Island, NY). DOX was purchased from JIAKE Chemical (Suzhou, China). Dialysis bags (molecular weight cut-off, $14 \mathrm{kDa}$ ) were from Solarbio Science \& Technology Co Ltd (Beijing, China). Cell counting kit-8 (CCK-8) was obtained from Dojindo Molecular Technologies (Kawasaki, Japan). Hoechst 33342, 3,3'-dioctadecyloxacarbocyanine perchlorate (DiO), and Coomassie Blue solution were purchased from Beyotime Biotechnology (Shanghai, China). FITC-labeled rabbit antimouse caspase-3 antibodies were purchased from AMEKO (Shanghai, China). Mouse monoclonal anti-human complement C3 polyclonal antibodies were purchased from Quidel (San Diego, CA, USA). Donkey anti-mouse, IRDye 800CWlabeled, secondary antibodies were from LI-COR Biosciences (Lincoln, NE, USA).

MC38 colon cancer cells were purchased from the Institute of Biochemistry and Cell Biology, Chinese Academy of Sciences. Human umbilical cord-derived MSCs were prepared in our laboratory.

\section{Quantitation of Iron Concentration by Iron Assay}

SPIO samples $(10 \mu \mathrm{L})$ were mixed with IRON Assay Kit Mix $(90 \mu \mathrm{L})$ and incubated at room temperature overnight to dissociate SPIO. Subsequently, the absorbance of the mixtures at $570 \mathrm{~nm}$ was determined. A series of diluted solutions of ferric chloride were used as standards for calibration (Figure S2).

\section{Loading of DOX on SPIO}

DOX was loaded on SPIO as follows: DOX dissolved in water $(1 \mathrm{mg} / \mathrm{mL})$ was added into a tube containing $100 \mu \mathrm{L}$ of SPIO (Fe: $1 \mathrm{mg} / \mathrm{mL}$ ). The mixture in the tube was wrapped in foil, to shield it from light, and incubated with shaking $(200 \mathrm{r} / \mathrm{min})$ at $37^{\circ} \mathrm{C}$ for at least $24 \mathrm{~h} .{ }^{29} \mathrm{DOX}$ SPIO NPs were separated by centrifugation at 100,000 g, using an Optima MAX-XP tabletop ultracentrifuge (Beckman Coulter, USA) for 5 min. The amount of DOX loaded was calculated as the difference between the amount of free DOX before and after conjugation. DOX concentrations were monitored by UV-Vis spectrometry at $498 \mathrm{~nm}$, using a NanoDrop Spectrophotometer (Thermo Scientific, Shanghai, China). Encapsulation efficiency (EE) was calculated using the following formula: ${ }^{30}$

$$
\begin{array}{r}
\mathrm{EE}(\%)=\text { Mass of DOX loaded on SPIO } \\
\text { / Initial mass of DOX } \times 100 \%
\end{array}
$$

\section{Drug Release in vitro}

To measure its drug release profile, $3 \mathrm{~mL}$ of DOX-SPIO solution was dialyzed against $20 \mathrm{~mL}$ of medium at $\mathrm{pH} 7.5$ 
(phosphate buffer) or $\mathrm{pH} 5.0$ (acetate buffer), with constant shaking $(60 \mathrm{rpm})$ at $37^{\circ} \mathrm{C}$. At various time intervals, $1 \mathrm{~mL}$ of dialysis buffer solution was removed for measurement and replaced by an equal volume of fresh medium. The amount of released DOX was determined by detecting the absorbance value at $498 \mathrm{~nm}$.

\section{Cell Culture}

MC38 cells were cultured in DMEM medium with 10\% FBS. MSCs were cultured in $\alpha$-MEM medium with $10 \%$ FBS and cells used for experiments had undergone less than 10 passages. All cells were kept in a humid cell incubator with $5 \% \mathrm{CO}_{2}$ atmosphere at $37^{\circ} \mathrm{C}$.

\section{Preparation of MSC Membrane-Derived Vesicles (MSVs)}

MSVs were prepared according to a published procedure, ${ }^{17}$ with slight modifications. After growing to almost $90 \%$ confluence, MSCs were detached using trypsin-EDTA, centrifuged, and resuspended in double distilled water $\left(\mathrm{DDH}_{2} \mathrm{O}\right)$ (containing $1 \mathrm{mM}$ phenylmethylsulfonyl fluoride) at $4^{\circ} \mathrm{C}$. After repeated freezing and thawing, the cells were gently sonicated on ice; the energy provided by sonication can separate MSCs into nuclei, cytoplasm, and cell membranes. Following centrifugation at $3200 \mathrm{~g}, 4^{\circ} \mathrm{C}$ for $10 \mathrm{~min}$, supernatants were further centrifuged at $15,000 \mathrm{~g}$ for $30 \mathrm{~min}$, to pellet cell membranes.

To prepare MSVs, we used a Mini-Extruder (Avanti Polar Lipids, Alabama, USA); prepared membrane fragments were extruded through 400 and $200 \mathrm{~nm}$ porous polycarbonate membranes in turn, 15 times each.

\section{Synthesis of DOX-SPIO@MSCs}

A co-extrusion method ${ }^{18}$ with slight modifications was used to synthesize DOX-SPIO@MSCs. MSVs (prepared as described above) were co-extruded with DOX-SPIO through a $200 \mathrm{~nm}$ polycarbonate porous membrane 10 times.

\section{NP-Tracking Analysis}

Mean NP hydrodynamic diameter was measured by NPtracking analysis using a NanoSight (NS300, Malvern Panalytical, Malvern, UK), fitted with a $638 \mathrm{~nm}$ laser reflector and an NS300 flow-cell top plate. Samples were diluted 1:1000 in $\mathrm{DDH}_{2} \mathrm{O}$ and immediately transferred to a $1 \mathrm{~mL}$ syringe by continuous constant speed pumping. All measurements were performed at room temperature with a vertical detection angle.

\section{Sodium Dodecyl Sulfate Polyacrylamide Gel Electrophoresis (SDS-PAGE) Protein Analysis}

The protein concentrations of cell membranes, DOX-SPIO @MSCs, and MSCs lysates were quantified by BCA protein assay. Then, samples with the same amount of total proteins were mixed well with $5 \times$ loading buffer and heated at $95^{\circ} \mathrm{C}$ for $5 \mathrm{~min}$ to denature the proteins. ${ }^{17}$ Protein contents were analyzed by $12.5 \%$ SDS-PAGE at $110 \mathrm{~V}$ for $3 \mathrm{~h}$. Gels were stained in Coomassie Blue solution for $2 \mathrm{~h}$, then destained in $\mathrm{DDH}_{2} \mathrm{O}$ overnight for imaging.

\section{Prussian Blue Staining}

MC38 cells $\left(1 \times 10^{5}\right)$ were seeded into each well of a 12well plate. After $12 \mathrm{~h}$ incubation, the cell culture medium was replaced with $1 \mathrm{~mL}$ fresh medium containing NPs. After another $4 \mathrm{~h}$ incubation, NPs were removed by washing the cells with PBS. Cells were then fixed in $4 \%$ paraformaldehyde, incubated with freshly prepared Perls' reagent $\left(10 \%\left(\mathrm{~K}_{4} \mathrm{Fe}(\mathrm{CN}) 6.3 \mathrm{H}_{2} \mathrm{O} / 20 \% \mathrm{HCl}, 1: 1 \text {, v/v }\right)^{19}\right.$ and subsequently imaged using an inverted microscope.

\section{Colocalization Study of DOX-SPIO@MSCs}

The cell membrane green fluorescent probe, DiO, was used to label MSVs before coating the DOX-SPIO core. DIOlabeled DOX-SPIO@MSCs NPs were then added to MC38 cells cultured in a 12-well plate. After $4 \mathrm{~h}$ incubation, the cells were washed and fixed in $4 \%$ paraformaldehyde for 30 min. Cell nuclei were stained with Hoechst 33342. Finally, the localization of DOX and MSVs in MC38 cells was visualized using an Olympus BX51 inverted fluorescence microscope (Tokyo, Japan).

\section{Cellular Uptake Study}

Flow cytometry (FCM) and fluorescence microscopy (FLM) were performed to investigate the cellular uptake behavior of NPs. ${ }^{30}$ For FCM, MC38 cells were seeded into 6-well plates. After $12 \mathrm{~h}$ incubation, cells were incubated with free form DOX, DOX-SPIO, or DOX-SPIO@MSCs for $30 \mathrm{~min}$ or $240 \mathrm{~min}$, with an equivalent DOX concentration of $50 \mu \mathrm{g} / \mathrm{mL}$. Thereafter, the cells were digested, resuspended in PBS, and analyzed using a CytoFLEX S Flow Cytometer (Beckman Coulter, USA).

For FLM, MC38 cells were seeded into 12-well plates and cultured overnight. Next, cells were incubated with different drugs for $240 \mathrm{~min}$, with the same DOX concentration used for 
FCM $(50 \mu \mathrm{g} / \mathrm{mL})$, then washed, fixed, and stained using Hoechst 33342. Finally, unbound dye was washed away with PBS and the cells observed using a fluorescence microscope.

\section{Tumor Cytotoxicity Evaluation}

In vitro cytotoxicity was assessed using the CCK- 8 cell viability assay. MC38 cells $\left(5 \times 10^{3}\right)$ were seeded into 96well plates and incubated overnight. Then, the medium in the wells was replaced with $200 \mu \mathrm{L}$ fresh medium containing free DOX, DOX-SPIO, or DOX-SPIO@MSCs, at different DOX concentrations, and the MC38 cells incubated for $48 \mathrm{~h}$. Next, the cells were washed with PBS three times and incubated with DMEM supplemented with $10 \%$ CCK- 8 for a further $2 \mathrm{~h}$. Finally, optical density (OD) at $450 \mathrm{~nm}$ was measured using a Multi-mode microplate reader (TECAN, Switzerland). Cell viability was calculated using the following equation: ${ }^{3}$

Cell viability $(\%)=$ OD test sample $/$ OD control $\times 100 \%$

\section{Hemolysis}

To study hemolysis, fresh sodium citrate anti-coagulated blood was donated by a healthy female volunteer. With reference to previous methods, ${ }^{20} 1 \mathrm{~mL}$ human fresh blood was centrifuged at $1000 \mathrm{~g}$ for $15 \mathrm{~min}$ to remove anti-coagulated plasma. The pellet was washed with cold PBS three times and resuspended in $5 \mathrm{~mL}$ PBS to prepare a red blood cell (RBC) suspension. PBS solutions $(0.8 \mathrm{~mL})$ with different concentrations of SPIO were mixed with $200 \mu \mathrm{L}$ RBC suspension; $0.8 \mathrm{~mL}$ water was used as a positive control yielding complete hemolysis and $0.8 \mathrm{~mL}$ PBS was added to $200 \mu \mathrm{L}$ RBC suspension as a negative control. After incubation in a water bath at $37^{\circ} \mathrm{C}$ for $3 \mathrm{~h}, \mathrm{RBC}$ suspensions were centrifuged at $1000 \mathrm{~g}$ for 15 min and the supernatants collected. Finally, absorbance values (A) of the released hemoglobin in the supernatants $(200 \mu \mathrm{L})$ were measured at $570 \mathrm{~nm}$. The percentage of hemolysis was calculated using the formula:

$$
\text { Hemolysis }(\%)=\frac{\text { Asample }- \text { Anegative }}{\text { Apositive }- \text { Anegative }} \times 100
$$

\section{In vitro Uptake of NPs by Human Leukocytes}

An in vitro leukocyte uptake experiment was conducted according to previous publications. ${ }^{21,22}$ Briefly, blood cells (RBCs, leukocytes, and platelets) were washed with $1 \%$ BSA-PBS solution to remove anti-coagulated plasma, then washed with PBS to remove BSA. NPs $(10 \mu \mathrm{L} ; \mathrm{Fe}, 1 \mathrm{mg} /$ $\mathrm{mL}$ ) were incubated with $30 \mu \mathrm{L}$ human serum at $37^{\circ} \mathrm{C}$ for $30 \mathrm{~min}$, then added to $100 \mu \mathrm{L}$ washed blood cells and incubated with leucocytes for a further $60 \mathrm{~min}$ at $500 \mathrm{rpm}$, $37^{\circ} \mathrm{C}$ using a Thermomixer (Thermo Scientific, Shanghai, China). Blood cells were then washed with PBS, to eliminate the uningested NPs, and leucocytes with internalized NPs isolated using a Mini MACS magnetic column (Miltenyi Biotec, USA). Magnetic white blood cells were then suspended in $200 \mu \mathrm{L}$ BSA-PBS for leucocyte counting.

\section{Analysis of Complement C3 Binding to Particles and Complement Activation Studies}

NPs $(\mathrm{Fe}, 1 \mathrm{mg} / \mathrm{mL})$ were incubated with human serum at a volume ratio of $1: 3$ at $37^{\circ} \mathrm{C}$. After incubation for 30 min, samples were centrifuged at 100,000 g. C5a ELISA kits (BlueGene Biotech, Shanghai, China) were used to measure complement activation in serum, according to the manufacturer's protocols. For complement $\mathrm{C} 3$ binding analysis, pellets were resuspended in PBS, and $1 \mu \mathrm{L}$ aliquots pipetted onto a nitrocellulose membrane (BioRad, USA). The membrane was blocked with 5\% skim milk in $1 \%$ PBS-T $\left(1 \times\right.$ PBS/Tween $\left.{ }^{\circledR} 20, \mathrm{v} / \mathrm{v}\right)$ for $1 \mathrm{~h}$ at room temperature, before incubating with the primary antibodies $(1: 1000)$ at $4^{\circ} \mathrm{C}$ overnight. The membrane was then washed with PBS- $T$ three times and finally incubated with IRDye800-conjugated secondary antibodies $(1: 15,000)$ for $2 \mathrm{~h}$ at room temperature. The dot-blot was scanned using an Odyssey infrared imager (Li-COR Biosciences, Lincoln, NE, USA).

\section{Animals and Tumor Model}

C57BL/6 female mice (6-8 weeks old) were purchased from Yi Si Laboratory Animal Center (Changchun, China). Animals were housed in a room at $25^{\circ} \mathrm{C}$ and $50 \%$ humidity, with a $12 \mathrm{hr}$ light/dark cycle. All animal procedures were approved by the Institutional Animal Care and Use Committee of Jilin University, where the study was performed. The guidelines of the committee were followed for the treatment of the animals.

A xenograft tumor model was established by subcutaneous injection of MC38 cells $\left(2 \times 10^{6}\right)$ into the shaved right rear flank of mice. Tumor volume was calculated as (tumor length) $\times(\text { tumor width })^{2} / 2{ }^{9}$ 


\section{In vivo Biodistribution Analysis}

When the MC38 xenograft tumor volumes increased to almost $250 \mathrm{~mm}^{3}$, tumor-bearing mice were intravenously injected with PBS, free DOX, DOX-SPIO, or DOX-SPIO @MSCs, at a DOX dosage of $10 \mathrm{mg} / \mathrm{kg}$. Mice were sacrificed at $24 \mathrm{~h}$ post-injection and major organs (heart, liver, spleen, lung, kidney, and tumor) harvested for ex vivo imaging and semi-quantitative biodistribution analysis. ${ }^{23-25}$

\section{Study of Therapeutics in Tumor-Bearing Mice}

When MC38 xenograft tumor volumes reached approximately $150 \mathrm{~mm}^{3}, \mathrm{C} 57 \mathrm{BL} / 6$ mice were randomly divided into four groups and intravenously injected with PBS, free DOX, DOXSPIO, or DOX-SPIO@MSCs. The injection (DOX, 4 mg/kg) was administered on days $0,2,4$, and 6 and tumor growth recorded every 2 days. After 16 days, all MC38 tumor-bearing mice were sacrificed. Major organs and tumors were collected and fixed in 4\% paraformaldehyde. A series of 4-mm sections were stained with hematoxylin-eosin (H\&E) and additional tumor sections stained by immunohistochemistry, terminal deoxynucleotidyl transferase-mediated nick end labeling (TUNEL), or caspase-3. Finally, stained tissue sections were observed using an inverted microscope.

\section{Statistical Analysis}

All statistical analyses were performed using Origin9 graphing and data analysis software (Originlab Northampton,
Massachusetts, USA). Data are presented as the mean \pm standard deviation, and differences between groups were assessed using the Student's $t$-test. Statistical significance was set at $\mathrm{p}<0.05$.

\section{Results and Discussion \\ Characterization of MSC \\ Membrane-Camouflaged NPs}

The synthesis strategy for DOX-SPIO@MSCs is diagrammatically illustrated in Figure 1, and consisted of three steps: preparing MSVs, loading DOX onto SPIO NPs, and coating DOX-SPIO with MSVs. TEM imaging clearly showed that DOX-SPIO@MSCs had an outer membrane shell of approximately $10 \mathrm{~nm}$ thickness and presented a typical core-shell structure (Figure $2 \mathrm{~A}$ ) ${ }^{26}$ the diameter of the particles was around $120 \mathrm{~nm}$. NTA revealed a mean hydrodynamic diameter of $125 \pm 1.3 \mathrm{~nm}$ and DOX-SPIO@MSCs had a relatively narrow particle size distribution $(90 \%$ of NPs had a diameter $<170 \mathrm{~nm}$ ) (Figure 2B).

Subsequently, we analyzed the protein contents of DOX-SPIO@MSCs by SDS-PAGE. Protein separation by PAGE showed that the protein band for DOX-SPIO@MSCs was similar to that of cell membranes, while it was slightly different from that of MSC lysates (Figure 2C). Further, analysis by SDS-PAGE revealed that the composition of membrane proteins was mostly retained through the preparation process, suggesting that the MSVs successfully coated the SPIO and retained most of the biological

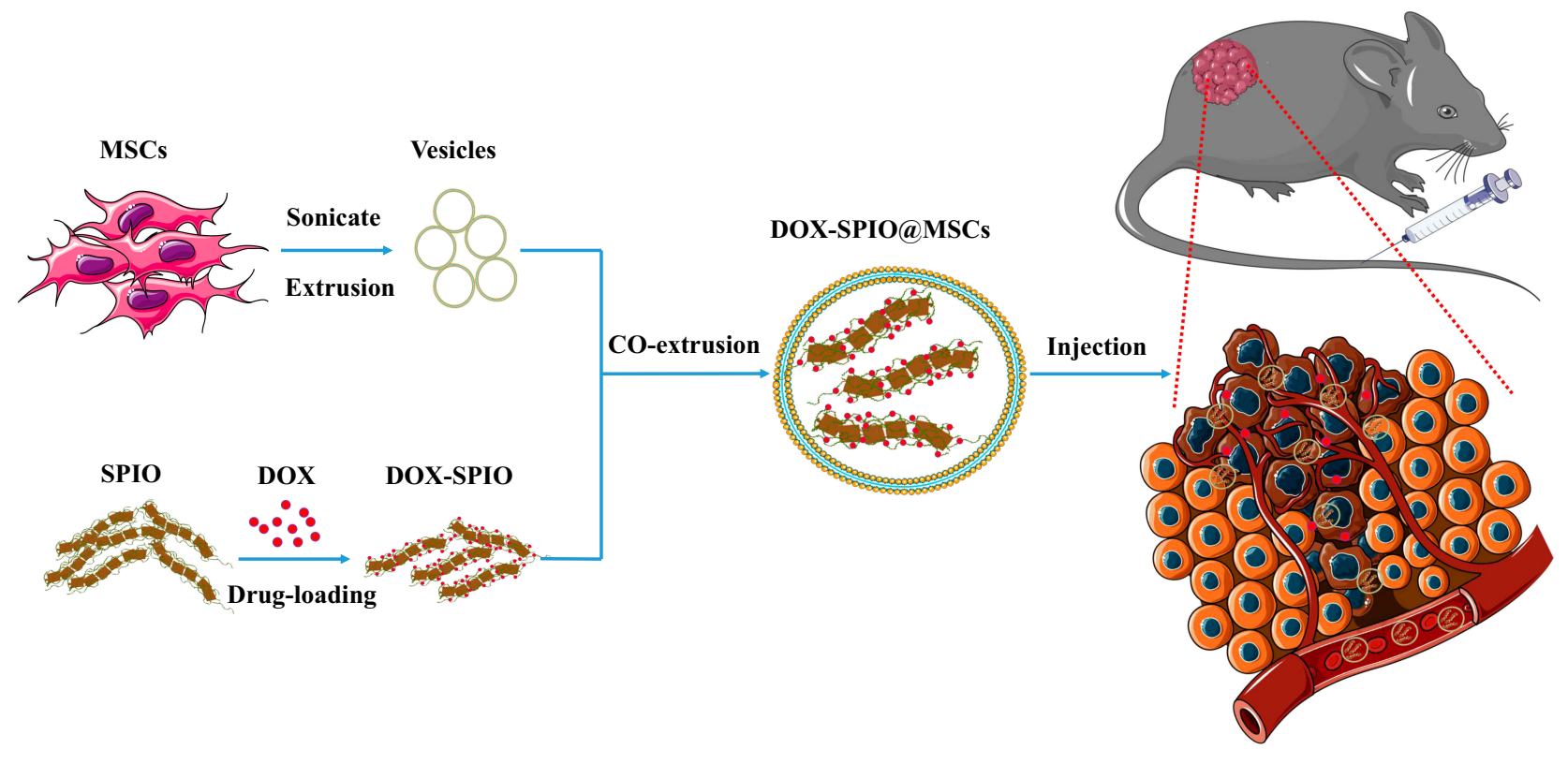

Figure I Schematic of the preparation of DOX-SPIO@MSCs and the tumor-targeting drug delivery in vivo. 
A

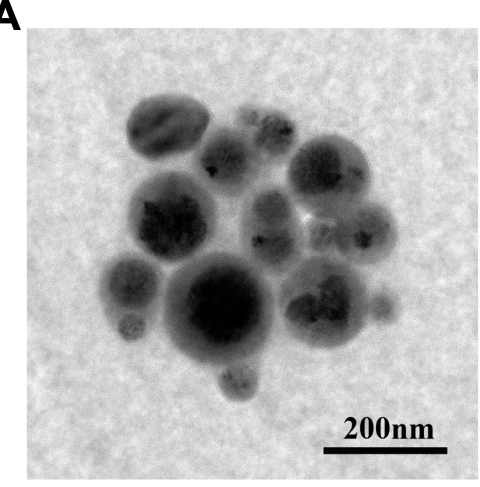

D

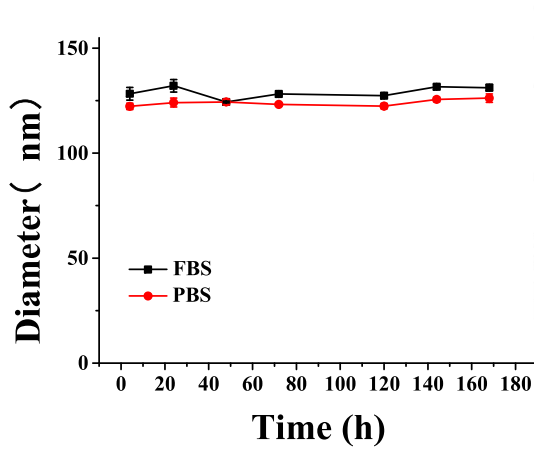

G
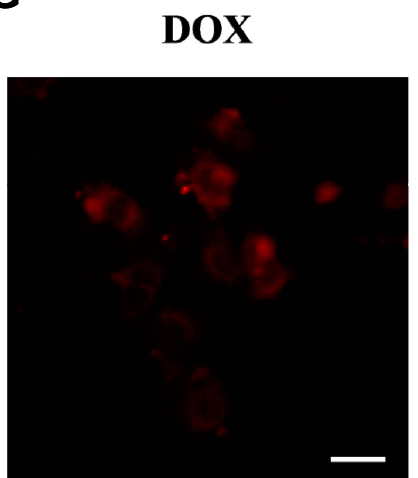

B

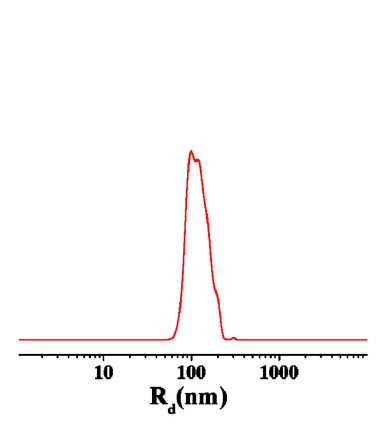

C

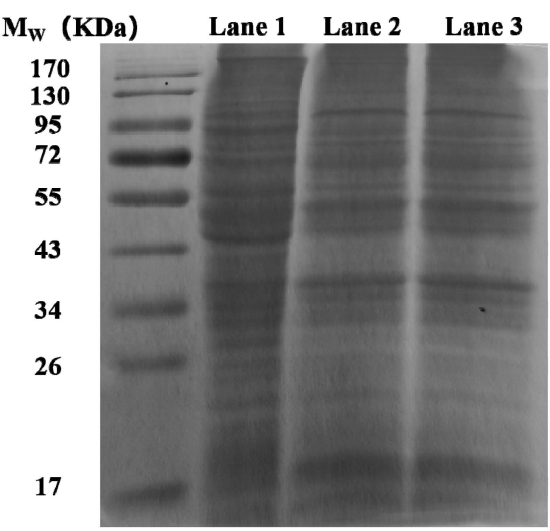

E

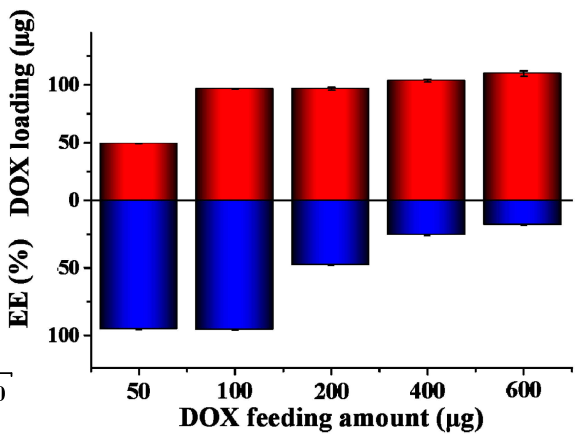

$\mathbf{F}$

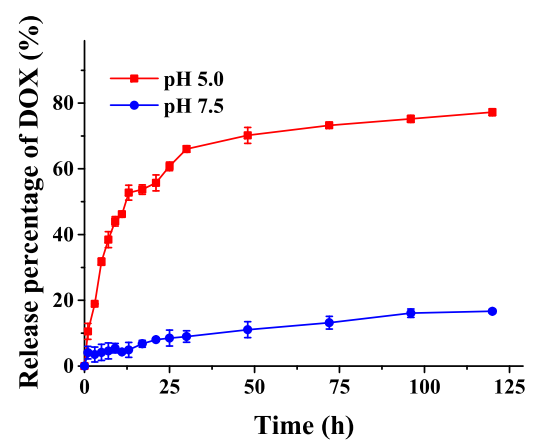

Figure 2 Characterization of DOX-SPIO@MSCs. (A) TEM image and (B) particle size distribution of DOX-SPIO@MSCs. (C) The SDS-PAGE images of the gel after Coomassie blue staining. Lane I: MSCs lysates, Lane 2: membrane vesicles, Lane 3: DOX-SPIO@MSCs. (D) The stability of DOX-SPIO@MSCs in FBS and PBS was evaluated by monitoring variation in particle size for different time intervals. (E) DOX loading amount and Encapsulation efficiency in 0.1 mg SPIO with different DOX feeding amounts. (F) The drug release profiles of the DOX-SPIO at different pH values. (G) Fluorescence microscope images of DOX-SPIO@MSCs illustrating colocalization of DOX@SPIO (DOX channel) and MSVs (DIO channel) after being internalized by MC38 cells. Scale bar $=40 \mu \mathrm{m}$.

functions of MSC membranes. ${ }^{27}$ To evaluate the long-term stability of DOX-SPIO@MSCs, fresh NPs were maintained in FBS and PBS at room temperature for 14 days, and the particle size monitored by NTA every day. As shown in Figure 2D, DOX-SPIO@MSCs exhibited good physiological stability, with minimal size changes over prolonged periods in both solutions. Fluorescence microscope imaging of MC38 cells incubated with DIO-labeled DOX-SPIO @ MSCs showed that red (DOX) and green (DIO) fluorescence were mostly co-localized (Figure 2G), revealing that DOX-SPIO@MSCs maintained their core-shell structure integrity after entering into cells. ${ }^{28}$ 


\section{Drug Encapsulation and Sustained Release of DOX-SPIO}

To investigate drug loading kinetics, encapsulated drug amounts in DOX-SPIO (SPIO:100 $\mu \mathrm{g}$ ), with a range of DOX feeding amounts, were measured. As shown in Figure 2E, drug loading (red column) was positively correlated with drug feeding amount. Overall, encapsulated amount increased with feeding amount; however, encapsulation efficiency (blue column) decreased when the feeding amount exceeded $100 \mu \mathrm{g}$.

Drug release behavior was investigated by immersing DOX-SPIO in solutions with two different $\mathrm{pH}$ values (Figure 2F), which mimicked conditions in the bloodstream (pH 7.5) and inside lysosomes (PH 5.0). At pH 5.0, a typical drug release curve was observed; that is, a relatively abrupt release during the first phase, with a slow and sustained second phase release. More than $60 \%$ of DOX was rapidly released in the first $36 \mathrm{~h}$, then the release rate gradually slowed down until $120 \mathrm{~h}$. In contrast, only $20 \%$ of DOX was released from DOX-SPIO during the entire drug release experiment at $\mathrm{pH}$ 7.5. These observations are attributable to lower $\mathrm{pH}$ values resulting in more rapid disintegration of the vesicles and re-protonation of amino groups. ${ }^{29,30}$ Most loaded drugs are expected to stay in NPs for extended periods of time after being injected into the blood circulation, with faster release on uptake into the lysosomes of cancer cells. pH-response release is characteristic of agents able to achieve tumor-targeting drug delivery.

\section{Internalization by MC38 Cells}

Efficient internalization by tumor cells is crucial for carriers of anti-tumor drugs. Prussian blue staining is a qualitative and visual approach for observing cellular iron distribution. As shown in Figure 3A, blue stained NPs were observed in the cytoplasm and gathered around the nuclei of MC38 cells, indicating that SPIO@MSCs were more readily taken up than SPIO.

FCM and FLM were performed to investigate the effect of MSC membrane coating on cellular uptake by MC38 Cells. Figure 3B(a) shows FCM histograms illustrating internalized DOX fluorescence in MC38 cells after 30 or $240 \mathrm{~min}$, clearly demonstrating that MSVs significantly improve the cellular uptake efficiency of DOX-SPIO after 240 min incubation. DOX mean fluorescence intensity was then measured by FCM (Figure 3B(b)). The fluorescence intensity from MC38 cells incubated with free DOX/DOX-SPIO@MSCs for $240 \mathrm{~min}$ was approximately 4.0 times that of cells incubated with DOX-SPIO. Fluorescent cell staining was used to further investigate cellular uptake behavior and study intracellular DOX distribution, by incubating MC38 cells with DOX-SPIO@MSCs, DOX-SPIO, or free DOX for $240 \mathrm{~min}$. The fluorescence intensity of cells treated with DOX-SPIO was significantly weaker than that of cells in the other groups, consistent with the results of FCM (Figure $3 C)$. In addition, DOX in free form predominately accumulated in the nuclei, while the majority of drug loading NPs remained in the cytoplasm. Free DOX accumulates in the nucleus because the molecules enter cells via the free diffusion pathway, and are quickly transferred to the nucleus, where they strongly bind to chromosomal DNA. ${ }^{31,32}$ In contrast, DOX-loaded NPs are taken up via endocytosis and accumulate in the lysosomes, where the nanocarriers degrade and drug release occurs. ${ }^{33}$ Overall, DOX-SPIO cellular uptake efficiency was significantly improved by surface modification with MSC membranes.

\section{Cytotoxicity Study}

Cytotoxicity against MC38 cells was evaluated using the CCK-8 assay. As shown in Figure 4A, cell viability decreased in response to increasing DOX concentration, with free DOX showing the strongest inhibition. This is due to direct penetration of the free drug into the nucleus, while the nanodrug is released slowly. Moreover, DOX-SPIO@MSCs presented better anti-tumor effects than DOX-SPIO at the same DOX concentrations, which could be because of higher cellular uptake of the membrane-coated NPs.

\section{Biological Compatibility}

The instability of the NPs in the bloodstream is regarded as the most serious limitation to their use as an intravenous drug-delivery platform. Therefore, we conducted hemolysis analysis to assess the blood compatibility of NPs. The hemolysis of RBCs incubated with different concentrations of SPIO is shown in Figure 4B. NPs exhibited no significant hemolytic effects. As shown in Figure S3, cell viability remained $>90 \%$ after incubation with SPIO at concentrations up to $100 \mu \mathrm{g} / \mathrm{mL}$ for $48 \mathrm{~h}$, indicating that SPIO has good biocompatibility.

NPs are subject to multiple interactions with the immune system, resulting in premature clearance, immune system activation, and toxicity. In vitro leukocyte uptake experiments showed that treatment with SPIO@MSCs resulted in a low percentage of magnetic white blood cells, indicating MSC membrane camouflage can reduce elimination of NPs by leukocytes (Figure 4C). Next, we 
A

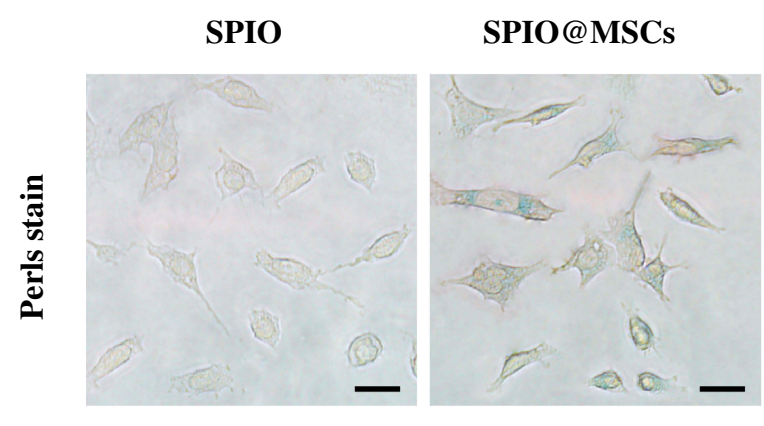

C

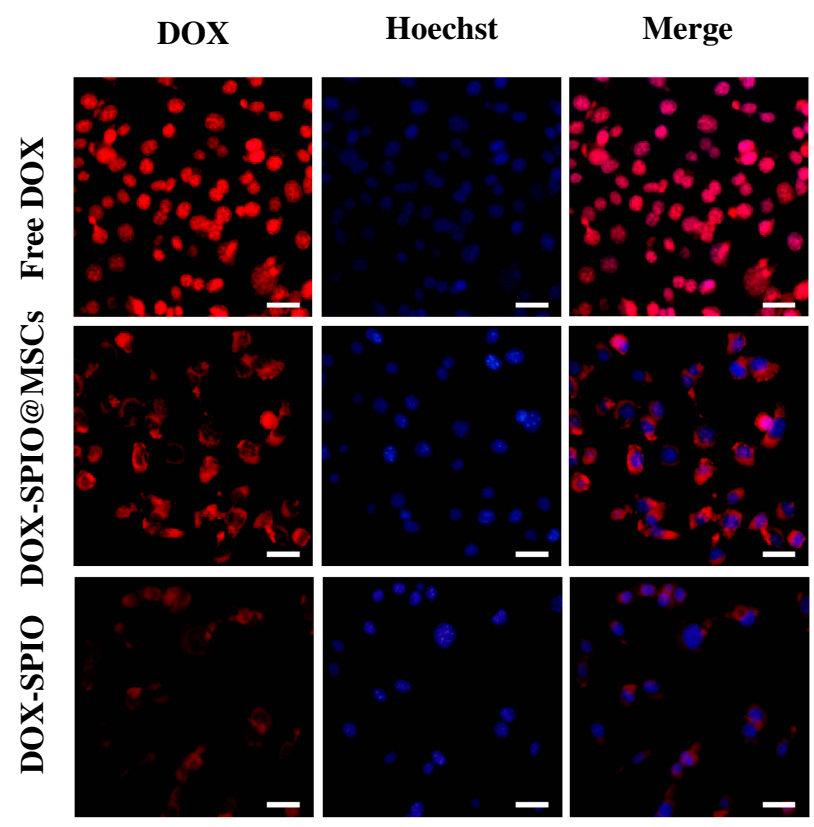

B

(a)
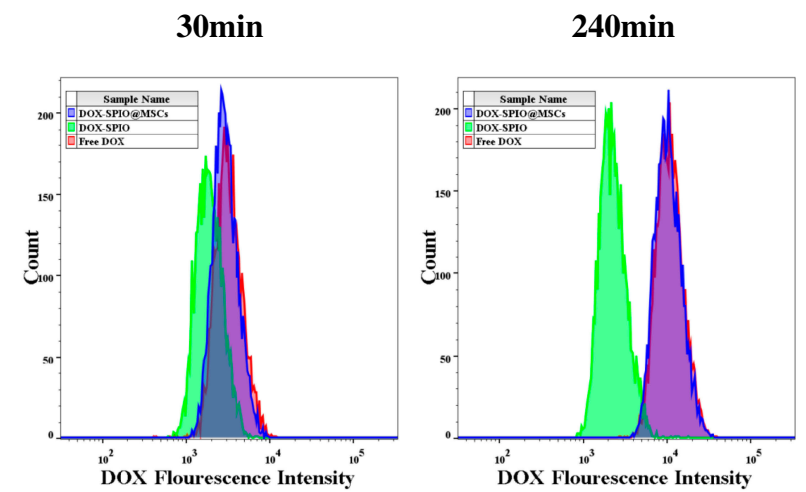

(b)

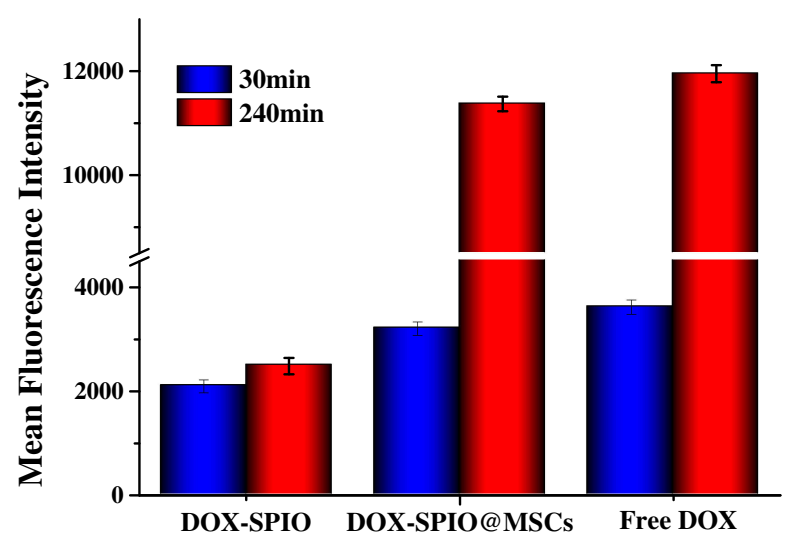

Figure 3 Intracellular distribution of NPs. (A) Prussian blue staining of MC38 cells after incubation with NPs for 4 h. Scale bar=20 $\mu$ m. (B) (a) Flow cytometry analysis of the MC38 cells treated with free DOX, DOX-SPIO and DOX-SPIO@MSCs for 30 and 240 min. (b) The DOX mean fluorescence intensity (MFI) of the MC38 cells. (C) Fluorescent staining images of the MC38 cells treated with different drugs for $240 \mathrm{~min}$. Scale bar $=40 \mu \mathrm{m}$.

investigated the $\mathrm{C} 3$ opsonization of NPs. There was a significant $(50 \%)$ decrease in $\mathrm{C} 3$ protein binding on SPIO@MSCs compared with SPIO (Figure 4D).

Human complement is mainly triggered by NPs via the alternative pathway, which induces initially deposited $\mathrm{C} 3 \mathrm{~b}$ associated with factor $\mathrm{B}$ to form the $\mathrm{AP}$ convertase, $\mathrm{C} 3 \mathrm{bBb}$, which cleaves additional $\mathrm{C} 3$ molecules. Complement activation further liberates two potent effector molecules, C3a and $\mathrm{C} 5 \mathrm{a}$, which are important for activation and recruitment of immune cells. ${ }^{34-36}$ To study complement activation, we measured the soluble marker, C5a, and demonstrated that
SPIO@MSCs blocked over $80 \%$ of C5a release, relative to SPIO (Figure 4E). Together, these data suggest that MSC membrane camouflage partially inhibits complementdependent uptake of NPs and release of proinflammatory factors.

\section{Ex vivo Organ Fluorescence Imaging and Biodistribution}

As shown in Figure 5A, in the free DOX-treated mouse group, fluorescence mainly accumulated in the major organs, which may cause severe systemic toxicity. In particular, 
A

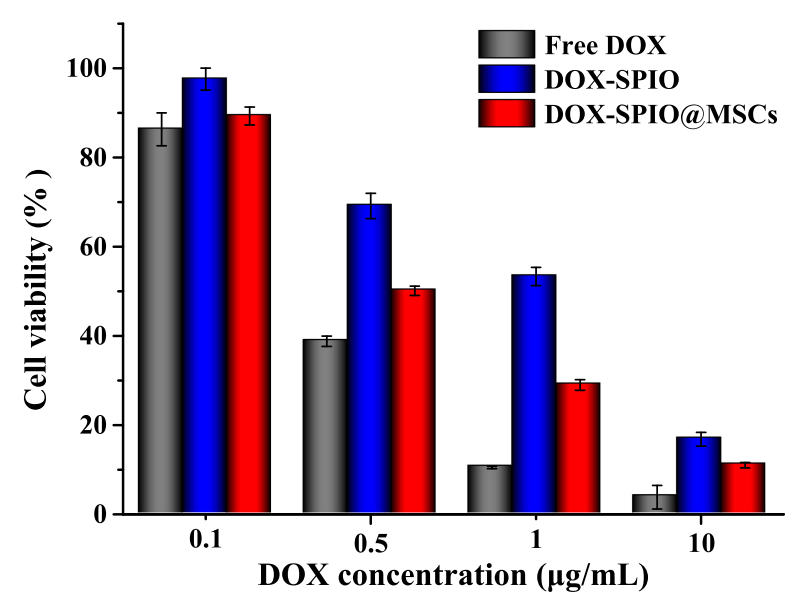

C

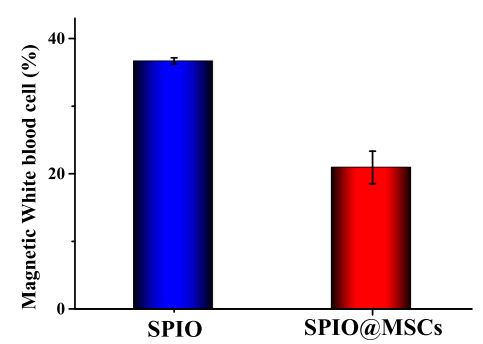

B

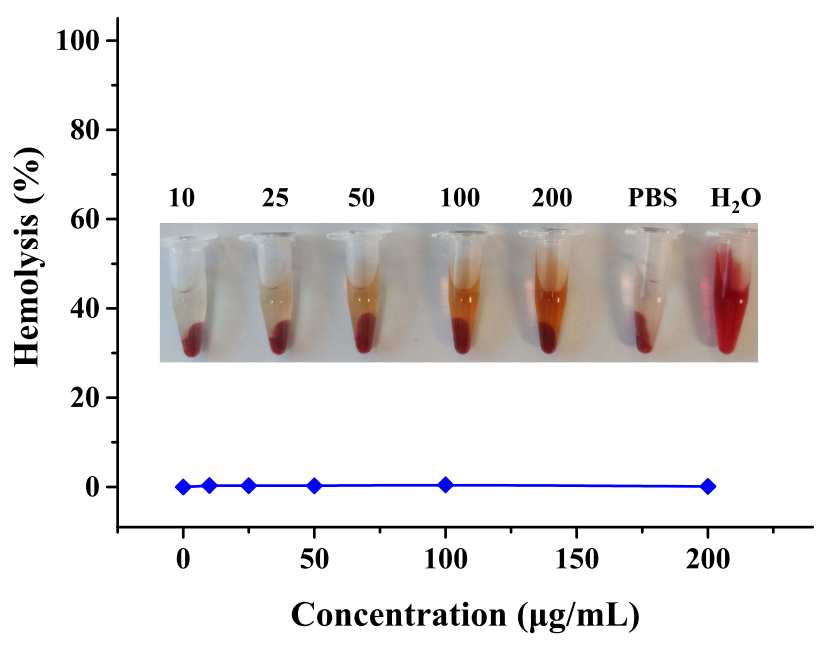

E

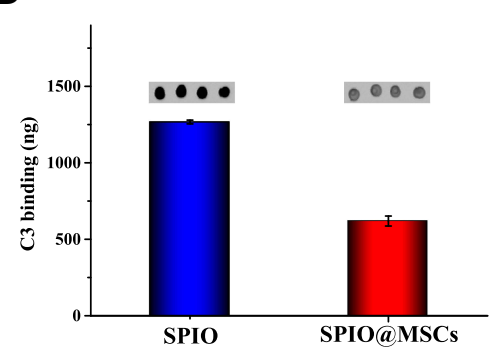

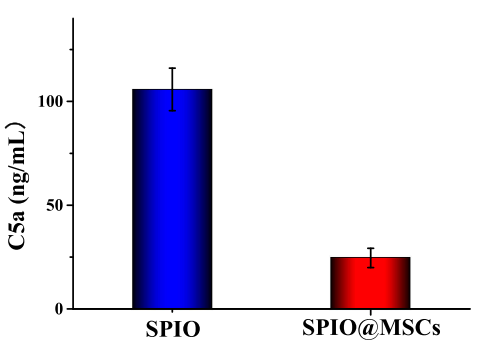

Figure 4 Biological responses in vitro (A) cytotoxicity of free DOX, DOX-SPIO and DOX-SPIO@MSCs against MC38 cells at different DOX concentrations. (B) Hemolysis study of SPIO with varied concentrations, using PBS as a negative control and water as a positive control. (C) Uptake of NPs by leukocytes from blood of a healthy donor in vitro. (D) Complement C3 opsonization of NPs. (E) Effect of NPs on complement activation and C5a generation.

A

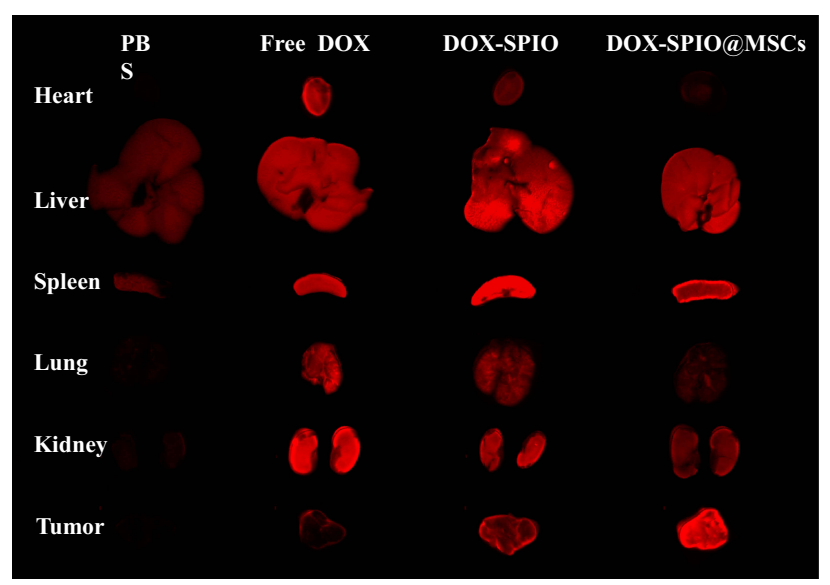

B

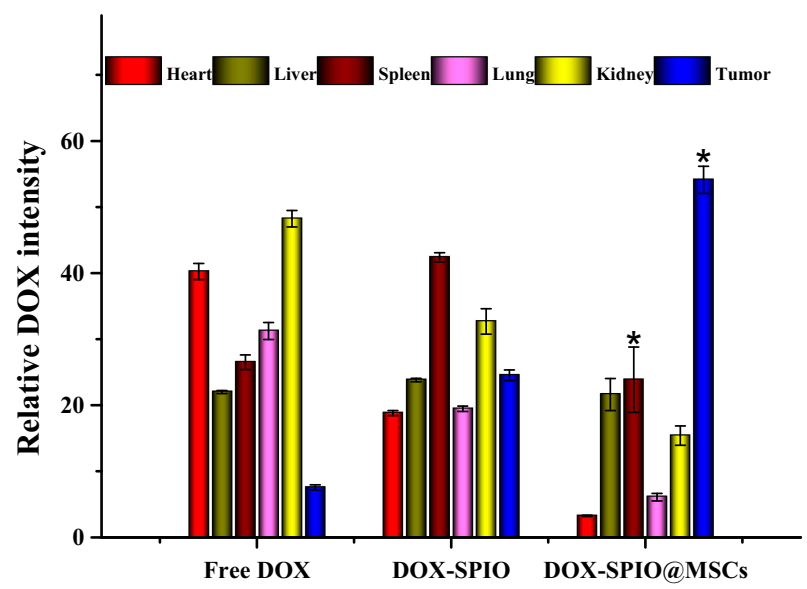

Figure 5 DOX biodistribution in tissues. Ex vivo DOX fluorescence images (A) and average DOX intensity (B) of main organs and tumors at $24 \mathrm{~h}$ post-injection of free DOX, DOX-SPIO, DOX-SPIO@MSCs. Considering the distraction of self-fluorescence of mice, we used the group PBS to set as background. *P<0.0I, versus DOX-SPIO $(n=3)$.

kidney exhibited strong fluorescence intensity, indicating that DOX was rapidly eliminated from the body in its free form. Further, spleens from mice in the DOX-SPIO@MSCs group exhibited weaker DOX fluorescence intensity compared with those from the DOX-SPIO group (Figure 5B), indicating that MSC membrane camouflage may avoid premature clearance 
of nanodrugs by organ-residing cells of the mononuclear phagocyte system, allowing NPs to reach target tissues. ${ }^{14}$ Furthermore, DOX fluorescence at tumor sites was stronger in mice treated with DOX-SPIO@MSCs than those receiving DOX-SPIO, confirming that MSC membrane camouflage can enhance tumor targeting. ${ }^{37,38}$

\section{In vivo Anti-Tumor Effects of DOX-SPIO@MSCs}

Measurement of tumor volumes in tumor-bearing mice revealed a significant tumor growth inhibition effect of

A

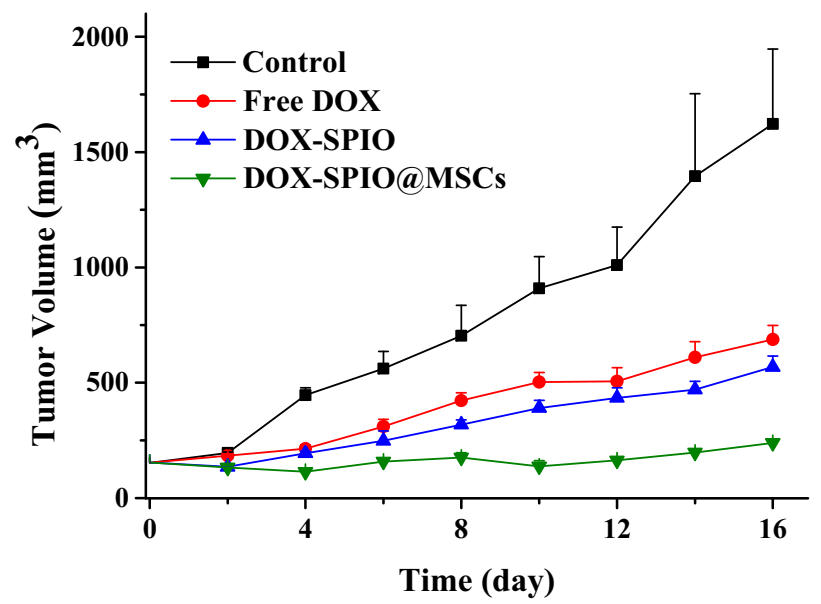

C

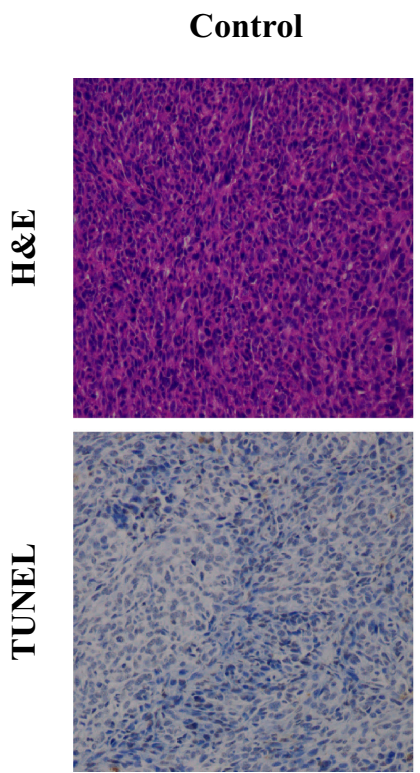

Free DOX
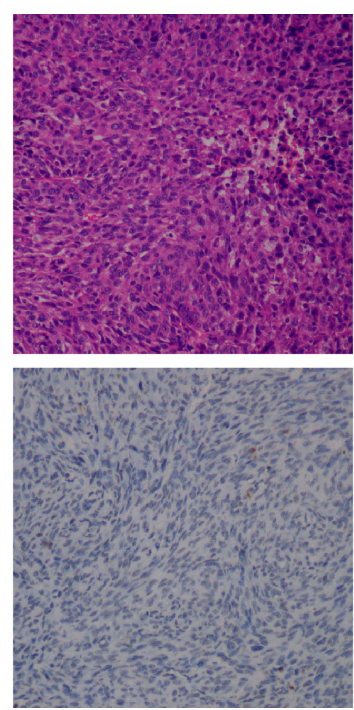

DOX-SPIO@MSCs after 16 days (Figure 6A). All tumors in DOX-SPIO@MSCs-treated mice were notably inhibited, with tumor volumes increasing extremely slowly throughout the 16 days of treatment. In contrast, mice in the PBS, free DOX, and DOX-SPIO groups showed increasing tumor volumes. At the end of the experiment, all tumors were dissected (Figure 6B) and the average tumor size from the DOX-SPIO@MSCs group was markedly smaller than that from the other three groups.

Further, in vivo toxicity was investigated by histological analysis. H\&E staining of major organs showed no

B

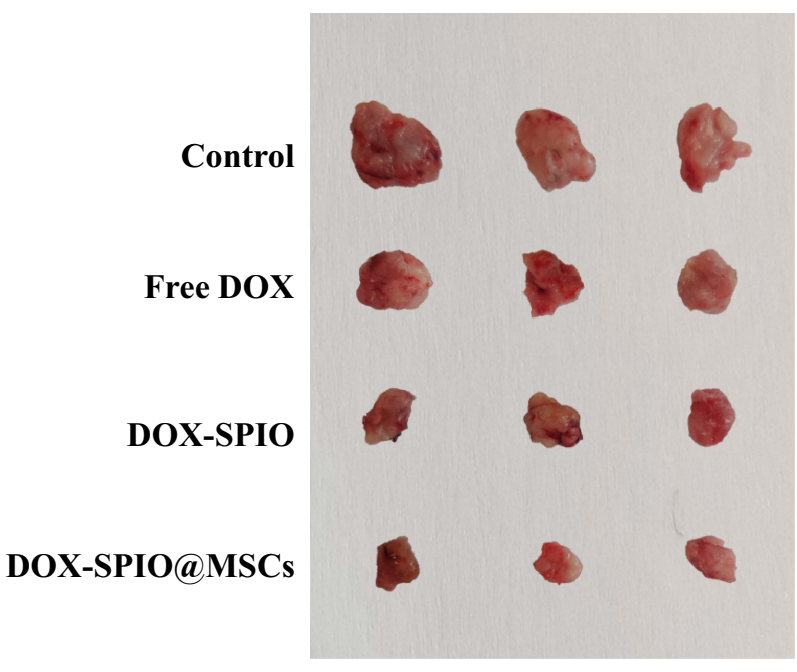

DOX-SPIO

DOX-SPIO@MSCs
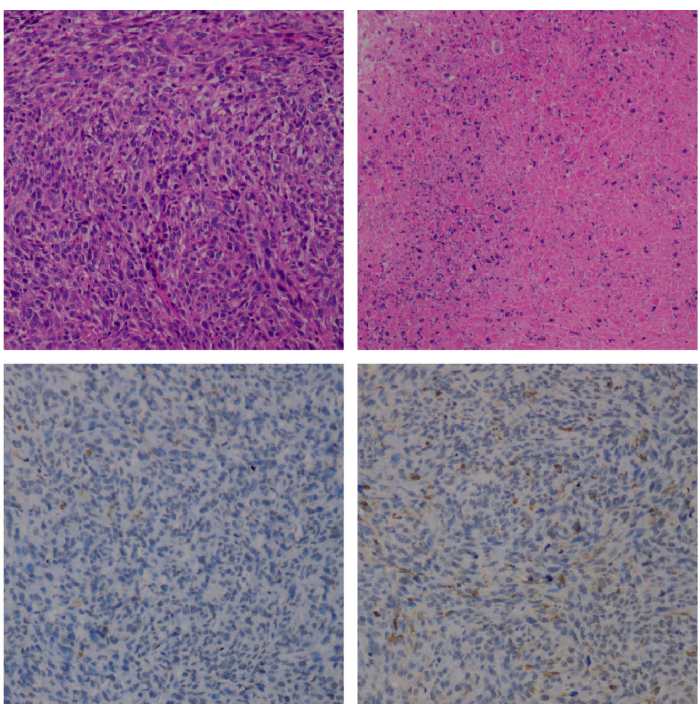

Figure 6 In vivo therapeutic effect. (A) Relative tumor volumes and curves of the tumor-bearing mice with different treatment. (B) Digital images show tumors harvested from different groups. (C) H\&E and TUNEL-stained tumor slice images (200x magnification). 
clear pathological variation in tissues from mice in the DOX-SPIO@MSCs group, implying that DOX-SPIO @MSCs do not exhibit obvious systematic toxicity (Figure S4). Moreover, analysis of tumor tissue samples from the DOX-SPIO@MSCs-treated group revealed pronounced morphological characteristics indicating apoptotic and necrotic regions, while tumor tissues from control group animals had normal morphology and nuclear structure. Furthermore, TUNEL assays showed apoptosis of a proportion of tumor cells in DOX-SPIO@MSCs-treated mice (Figure 6C), with consistent results for caspase-3 staining (Figure S5). Overall, MSC membranes effectively camouflaged NPs, improving tumor targeting, and exhibited mild systemic toxicity.

\section{Conclusion}

By coating nanodrugs with MSC membranes, we have developed a novel medicine delivery platform. DOXSPIO@MSCs possess the biological functions of MSC membranes, allowing them to achieve high intracellular delivery, prolonging serum half-life, and efficiently targeting tumors. Compared with free DOX and DOX-SPIO, the anti-tumor therapeutic efficiency of DOX-SPIO@MSCs was markedly enhanced, while use of these NPs reduced the systemic toxicity of DOX. In summary, these results demonstrate the great potential of DOX-SPIO@MSCs as a promising biomimetic NPs platform for cancer treatment in the future.

\section{Ethics and Consent Statement}

We obtained all written informed consent from objects and Ethical approval for this investigation was approved by the Research Ethics Committee, China-Japan Union Hospital (approval No.2019-NSFC-023). The study was carried out in accordance with the Declaration of Helsinki.

\section{Disclosure}

Professor Fangfang Chen reports grants from the National Natural Science Foundation of China (Grant No. 31771093), grants from the Project of International Collaboration of Jilin Province (No.201180414085GH), grants from the Fundamental Research Funds for the Central Universities, the Program for JLU Science and Technology Innovative Research Team (2017TD-27, 2019TD-36), during the conduct of the study. The authors declare no other conflict of interest in this work.

\section{References}

1. Das D, Rameshbabu AP, Ghosh P, Patra P, Dhara S, Pal S. Biocompatible nanogel derived from functionalized dextrin for targeted delivery of doxorubicin hydrochloride to MG 63 cancer cells. Carbohydr Polym. 2017;171:27-38. doi:10.1016/j.carbpol.2017.04.068

2. Akhtar S, Suriya Rehman S, Almessiere MA, et al. Synthesis of $\mathrm{Mn} 0.5 \mathrm{Zn} 0.5 \mathrm{SmxEuxFe} 1.8-2 \mathrm{xO} 4$ nanoparticles via the hydrothermal approach induced anti-cancer and anti-bacterial activities. Nanomaterials. 2019;9(11):1635. doi:10.3390/nano9111635

3. El Rayes SM, Ahmed Aboelmagd A, Gomaa, et al. Convenient synthesis and anticancer activity of methyl 2-[3-(3-Phenylquinoxalin-2-ylsulfanyl)propanamido]alkanoates and N-Alkyl 3-((3-Phenyl-quinoxalin-2-yl)sulfanyl)propanamides. ACS Omega. 2019;4(20):18555-18566. doi:10.1021/acsomega.9b02320

4. Asiri SM, Khan FA, Bozkurt A. Delivery of conjugated silicon dioxide nanoparticles show strong anti-proliferative activities. Appl Biochem Biotechnol. 2019;189(3):760-773. doi:10.1007/s12010-01903030-0

5. Pasto A, Giordano F, Evangelopoulos $M$, et al. Cell membrane protein functionalization of nanoparticles as a new tumor-targeting strategy. Clin Trans Med. 2019;8(1):8. doi:10.1186/s40169-0190224-y

6. Anchordoquy TJ, Barenholz Y, Boraschi D, et al. Mechanisms and barriers in cancer nanomedicine: addressing challenges, looking for solutions. ACS Nano. 2017;11(1):12-18. doi:10.1021/acsnano.6b 08244

7. Yang N, Ding Y, Zhang Y, et al. Surface functionalization of polymeric nanoparticles with umbilical cord-derived mesenchymal stem cell membrane for tumor-targeted therapy. ACS Appl Mater Interfaces. 2018;10(27):22963-22973. doi:10.1021/acsami.8b05363

8. Chena M, Chen M, He J. Cancer cell membrane cloaking nanoparticles for targeted co-delivery of doxorubicin and PD-L1 siRNA. Artif Cells Nanomed Biotechnol. 2019;47(1):1635-1641. doi:10.1080/ 21691401.2019.1608219

9. Zhang W, Yu M, Xi Z, et al. Cancer cell membrane-camouflaged nanorods with endoplasmic reticulum targeting for improved antitumor therapy. ACS Appl Mater Interfaces. 2019;11(50):46614-46625. doi:10.1021/acsami.9b18388

10. Minliang W, Wenjun L, Tianxiao M, et al. Cell membrane camouflaged nanoparticles: a new biomimetic platform for cancer photothermal therapy. Int J Nanomed. 2019;14:4431-4448. doi:10.2147/ IJN.S200284

11. Vijayan V, Uthaman S, Park I-K. Cell membrane-camouflaged nanoparticles: a promising biomimetic strategy for cancer theragnostics. Polymers. 2018;10(9):983. doi:10.3390/polym10090983

12. Patel DK, Jin B, Dutta SD, Lim KT. Osteogenic potential of human mesenchymal stem cells on eggshells-derived hydroxyapatite nanoparticles for tissue engineering:. J Biomed Mater Res B Appl Biomater. 2019. doi:10.1002/jbm.b.34536

13. Shin TH, Lee DY, Ketebo AA, et al. Silica-coated magnetic nanoparticles decrease human bone marrow-derived mesenchymal stem cell migratory activity by reducing membrane fluidity and impairing focal adhesion. Nanomaterials. 2019;9(10):1475. doi:10.3390/ nano9101475

14. Narain A, Asawa S, Chhabria V, et al. Cell membrane coated nanoparticles: next-generation therapeutics. Nanomedicine. 2017;12 (21):2677-2692. doi:10.2217/nnm-2017-0225

15. Wu HH, Zhou Y, Tabata Y, Gao JQ. Mesenchymal stem cell-based drug delivery strategy: from cells to biomimetic. J Control Release. 2019;28(294):102-113. doi:10.1016/j.jconrel.2018.12.019

16. Letko Khait N, Malkah N, Kaneti G, et al. Radiolabeling of cell membrane-based nano-vesicles with 14C-linoleic acid for robust and sensitive quantification of their biodistribution. $J$ Control Release. 2019;293:215-223. doi:10.1016/j.jconrel.2018.12.005 
17. Gao C, Lin Z, He Q, et al. Stem cell membrane-coated nanogels for highly efficient in vivo tumor targeted drug delivery. Small. 2016;12 (30):4056-4062. doi:10.1002/smL1.201600624

18. Mu XP, Li J, Jiang JL, et al. siRNA delivery with stem cell membrane-coated magnetic nanoparticles for imaging-guided photothermal therapy and gene therapy. ACS BIOMAT SCI ENG. 2018;4 (10):3895-3905. doi:10.1021/acsbiomaterials.8b00858

19. Zhu XM, Yuan J, Leung KC, et al. Hollow superparamagnetic iron oxide nanoshells as a hydrophobic anticancer drug carrier: intracellular pH-dependent drug release and enhanced cytotoxicity. Nanoscale. 2012;4(18):5744-5754. doi:10.1039/c2nr30960b

20. Liu X, Mo Y, Liu X, et al. Synthesis, characterisation and preliminary investigation of the haemocompatibility of polyethyleneimine-grafted carboxymethyl chitosan for gene delivery. Mater Sci Eng C Mater Biol Appl. 2016;62:173-182. doi:10.1016/j.msec.2016.01.050

21. Inturi S, Wang G, Chen F, et al. Modulatory role of surface coating of superparamagnetic iron oxide nanoworms in complement opsonization and leukocyte uptake. ACS Nano. 2015;9(11):10758-10768. doi:10.1021/acsnano.5b05061

22. Wang G, Griffin J, Inturi S, et al. In vitro and in vivo differences in murine third complement component (C3) opsonization and macrophage/leukocyte responses to antibody-functionalized iron oxide nanoworms. Front Immunol. 2017;8:151. doi:10.3389/fimmu. 2017.00151

23. Gao F, Zhang C, Qiu WX, et al. PD-1 blockade for improving the antitumor efficiency of polymer-doxorubicin nanoprodrug. Small. 2018;14(37)::e1802403. doi:10.1002/smll.201802403

24. Song S, Chong Y, Fu H, et al. HP- $\beta$-CD functionalized Fe3O4/CNPsbased theranostic nanoplatform for $\mathrm{ph} / \mathrm{nir}$ responsive drug release and MR/NIRFL imaging-guided synergetic chemo/photothermal therapy of tumor. ACS Appl Mater Interfaces. 2018;10(40):33867-33878. doi:10.1021/acsami.8b09999

25. Zhang L, Qin Y, Zhang Z, et al. Dual pH/reduction-responsive hybrid polymeric micelles for targeted chemo-photothermal combination therapy. Acta Biomater. 2018;75:371-385. doi:10.1016/j.actbio. 2018.05.026

26. Fang RH, Kroll AV, Gao W, et al. Cell membrane coating nanotechnology. Adv Mater. 2018;30(23):e1706759. doi:10.1002/ adma.201706759

27. Lai P-Y, Huang R-Y, Lin S-Y, et al. Biomimetic stem cell membrane-camouflaged iron oxide nanoparticles for theranostic applications. RSC $A d v$. 2015;5(119):98222-98230. doi:10.1039/ c5ra17447c
28. Peng L-H, Zhang Y-H, Han L-J, et al. Cell membrane capsules for encapsulation of chemotherapeutic and cancer cell targeting in vivo. ACS Appl Mater Interfaces. 2015;7(33):18628-18637. doi:10.1021/ acsami.5b05065

29. Yang X, Grailer JJ, Rowland IJ, et al. Multifunctional SPIO/ DOX-loaded wormlike polymer vesicles for cancer therapy and MR imaging. Biomaterials. 2010;31(34):9065-9073. doi:10.1016/j. biomaterials.2010.08.039

30. Li X, Li N, Sidlauskas K, et al. Doxorubicin-loaded dextran-modified goldmag nanoparticles for targeting hepatocellular carcinoma. J Biomed Nanotechnol. 2018;14(6):1135-1146. doi:10.1166/ jbn.2018.2547

31. Zhao Y, Huan M-L, Liu M, et al. Doxorubicin and resveratrol co-delivery nanoparticle to overcome doxorubicin resistance. Sci Rep. 2016;6(1):35267. doi:10.1038/srep35267

32. Khan FA, Akhtar S, Almohazey D, et al. Targeted delivery of poly (methyl methacrylate) particles in colon cancer cells selectively attenuates cancer cell proliferation. Artif Cells Nanomed Biotechnol. 2019;47(1):1533-1542. doi:10.1080/21691401.2019.1577886

33. arhane Z, Bonnier F, Byrne HJ. Monitoring doxorubicin cellular uptake and trafficking using in vitro Raman microspectroscopy: short and long time exposure effects on lung cancer cell lines. Anal Bioanal Chem. 2017;409(5):1333-1346. doi:10.1007/s00216-0160065-0

34. Chen F, Wang G, Griffin J, et al. Complement proteins bind to nanoparticle protein corona and undergo dynamic exchange in vivo. NatNanotechnol. 2017;12(4):387-393. doi:10.1038/nnano.2016.269

35. Simberg D. Iron oxide nanoparticles and the mechanisms of immune recognition of nanomedicines. Nanomedicine. 2016;11(7):741-743. doi:10.2217/nnm.16.30

36. Banda NK, Mehta G, Chao Y, et al. Mechanisms of complement activation by dextran-coated superparamagnetic iron oxide (SPIO) nanoworms in mouse versus human serum. Part Fibre Toxicol. 2014;11(1):64. doi:10.1186/s12989-014-0064-2

37. Ma K, Fu D, Liu Y, et al. Cancer cell targeting, controlled drug release and intracellular fate of biomimetic membrane-encapsulated drug-loaded nano-graphene oxide nanohybrids. J Mater Chem B. 2018;6(31):5080-5090. doi:10.1039/C8TB00804C

38. Luk BT, Zhang L. Cell membrane-camouflaged nanoparticles for drug delivery: cell membrane-camouflaged nanoparticles for drug delivery. J Control Release. 2015;220:600-607. doi:10.1016/j. jconrel.2015.07.019
International Journal of Nanomedicine

\section{Publish your work in this journal}

The International Journal of Nanomedicine is an international, peerreviewed journal focusing on the application of nanotechnology in diagnostics, therapeutics, and drug delivery systems throughout the biomedical field. This journal is indexed on PubMed Central, MedLine, CAS, SciSearch ${ }^{\mathbb{}}$, Current Contents ${ }^{\mathbb{R}} /$ Clinical Medicine, $^{-}$

\section{Dovepress}

Journal Citation Reports/Science Edition, EMBase, Scopus and the Elsevier Bibliographic databases. The manuscript management system is completely online and includes a very quick and fair peer-review system, which is all easy to use. Visit http://www.dovepress.com/ testimonials.php to read real quotes from published authors. 